\title{
Nanosheet Formation in Hyperswollen Lyotropic Lamellar Phases
}

\author{
Yoshiaki Uchida, ${ }^{*, \$}$, Takuma Nishizawa, ${ }^{*}$ Takeru Omiya, ${ }^{*}$ Yuichiro Hirota, ${ }^{*}$ and \\ Norikazu Nishiyama* \\ ॠGraduate School of Engineering Science, Osaka University, 1-3 Machikaneyama-cho, Toyonaka, Osaka \\ 560-8531, Japan \\ ${ }^{\S}$ PRESTO, Japan Science and Technology Agency (JST), Kawaguchi, Saitama 332-0012, Japan
}

\section{Supporting Information}

\section{Derivation of layer spacings}

From a selective reflection peak, the corresponding layer spacing can be calculated by Bragg equation

$$
d=\frac{\lambda}{2 n \cos \theta}
$$

where $d$ is layer spacing, $\lambda$ is selective reflection peak wavelength, $n$ is refractive index of water (1.33), and $\theta$ is incident angle $\left(12^{\circ}\right)$. 


\section{Figures}

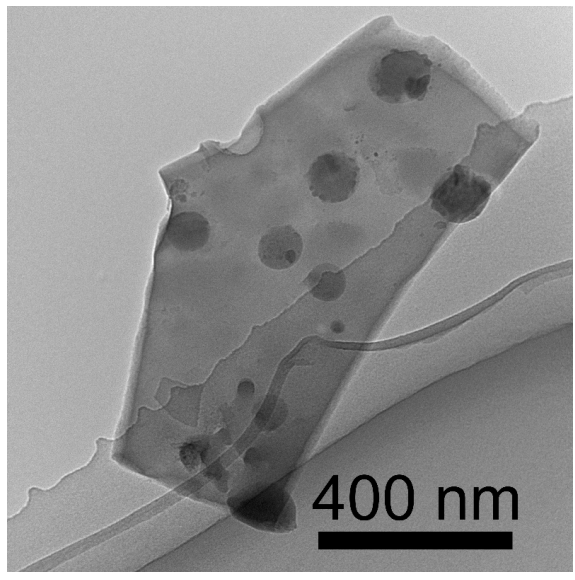

Figure S1. TEM photograph of one of the synthesized carbon nanosheets.
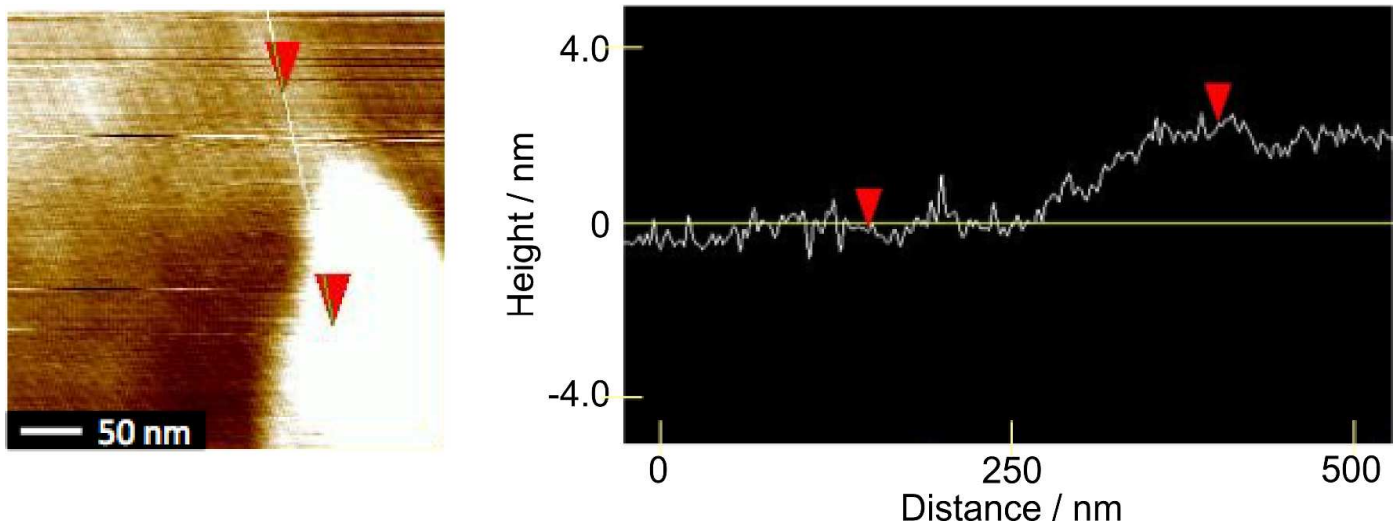

Figure S2. AFM photograph and cross section of one of the synthesized carbon nanosheets. 